\title{
Measures for the Ontological Relations in Enterprise
}

\author{
Sabria Hadj Tayeb \\ Département Mathématique/ Informatique \\ Université des Sciences et de la Technologie d'Oran Mohamed Boudiaf, USTO-MB, \\ BP1505, El M'naouar Oran, 31000, Algérie \\ Email: sabria.hadjtayeb@univ-usto.dz \\ Myriam Noureddine \\ Département Mathématique/ Informatique \\ Université des Sciences et de la Technologie d'Oran Mohamed Boudiaf, USTO-MB, BP1505, \\ El M'naouar Oran, 31000, Algérie \\ Email: myriam.noureddine@univ-usto.dz
}

Received: 17 January 2017; Accepted: 22 August 2017; Published: 08 September 2017

\begin{abstract}
In order to improve a system performance, it is significant to estimate the exchange rate of relationships between components of the system, in particular when the considered system is production or service companies. Indeed, bad and inappropriate relationships can generate dysfunctions, slowdowns or, more generally, loss of performance in enterprise leading to a decline in growth and competitiveness.

Because of the heterogeneity of information and data, it is necessary to modeling relationships and ontologies are currently among the most evoked models in knowledge engineering. The aim is to define structured vocabularies, bringing together useful concepts of a domain and their relationships thus serving to organize, exchange information in an unambiguous way. Ontologies are widely applied to ensure semantic interoperability describing the enterprise structure and the exchange rate of existing relationships can be valued through their degree of effectiveness.

This paper presents measures for the ontological relations in the enterprise. Our approach aims first to extract the set of relationships from an ontology previously created, then classify these relations, according to two types giving a weighting to calculate their degree of effectiveness. The implementation process is proposed on the local enterprise of steel wire drawing processing, giving degree of effectiveness for existing relationships. A sensitivity analysis is done to compare and interpret the different results.
\end{abstract}

Index Terms-Enterprise, Performance, Ontology, Effectiveness.

\section{INTRODUCTION}

To overcome the problems of heterogeneity due development and disparity data, approaches have emerged and are thriving as ontologies. Ontologies are formal systems whose objective is to represent the knowledge of a domain by means of concepts, defined and structured. The ontological representation of knowledge ensures the maintenance of the coherence and integrity of the system as well as the evolution of the representation without changing the structure while improving the exploitation of the information resources. The ontologies are also widely used to ensure semantic interoperability since they allow presenting the company structure and the existing relationships, allowing to calculate the effectiveness or to optimize relations. In order to improve enterprise performance, it is important to estimate the rate of exchange relationships.

This article presents measures for the ontological relations in enterprise to estimate this rate following their effectiveness degree.

The paper remainder is organized as follows: In the second section, we introduce the notion of effectiveness of semantic relations. In the third section, the proposed approach is exhibited. The fourth section describes the application of the approach to an enterprise dedicated to the transformation steel, specialized in welded mesh. The result given percentage allows comparing different results

\section{EFFECTIVENESS OF ONTOLOGICAL RELATIONSHIPS}

In this section, we present the effectiveness of ontological relationships. The relationships within the enterprise

\section{A. The relationships within the enterprise}

The relationRel can be defined as the relationship between two concepts A and B. We note "Rel Sem" $_{\text {" the }}$ semantic relationship that highlights the significance of links between concepts.

The use of semantic relations will be used to ensure interoperability. This could be seen as an overall requirement that enterprises in partnership situation must 
take on. Interoperability is defined as the ability of a collection of communicating entities to share specified information and operate on that information according to a shared operational semantics in order to achieve a specified purpose in a given context [1] [2].

\section{B. Measuring effectiveness}

According to Moigne [3], the effectiveness is to report the result of an action for which it is based. A criterion is not the question how much it costs but the question of whether the actor has actually done what he wanted to do. In our context, the effectiveness of semantic relationships is to assess the relationship by contribution to their goals.

Based on the work of [4], measuring effectiveness of semantic relations is calculated by splitting the number (Nbr) of semantic relations by the total number (NbrT) of semantic relations. Therefore, we note the measurement of effectiveness of semantic relations $E f f_{\text {RelSem }}$ by the formula (1):

$$
E f f_{\text {RelSem }}=\frac{\mathrm{NbrRel}_{\mathrm{Sem}}}{\operatorname{NbrTRel}_{\mathrm{Sem}}}
$$

\section{PROPOSED APPROACH}

Our approach aims first to create an ontology in order to extract the semantic relations and then to calculate the degree of effectiveness of the extracted semantic relations.

\section{A. Ontological approach}

To ensure semantic interoperability, fundamentally, the information exchanged must first be formally described and the use of ontologies is the most appropriate way. Several definitions have been proposed to define ontologies and the definition of Gruber [5] in 1993 remains the definition of reference "An ontology is a formal and explicit specification of a conceptualization".

This definition meets the essential features of an ontology:

- Conceptualization: According to Gruber [5], a conceptualization is "An abstract, simplified view of the world". That said, an abstract and simplified view of the world that we wish to represent for a given purpose.

- $\quad$ Formal specification of a conceptualization refers to the ability of ontology to transpose conceptualization into a language that can be interpreted by the machine.

- Explicit: The type of concepts applied and the constraints on their use are explicitly defined. In general, ontologies describe the structure and semantics of data. They allow users to organize information on concepts, attributes and relationships, according to a domain ontology that allows a meta-knowledge representation. Ontologies [6] [7] thus provide a common language defining the meaning of terms and the relationships between them.

\section{B. Elements of ontology}

The ontologies [8] allow to represent the knowledge and to manipulate them automatically while keeping their semantics. This knowledge is presented in the taxonomy of the concepts, each with their attributes while describing the relationships between these concepts.

An entire ontology $\mathrm{O}$ is defined by the structure of the form [9]:

$$
O:=(\mathrm{C}, \leq \mathrm{c}, \mathrm{R}, \leq \mathrm{R}, \sigma \mathrm{R}, \mathrm{A}, \sigma \mathrm{A}, \mathrm{T})
$$

With:

$C$ : A set of concepts;

$R$ : A set of relations;

$A$ : A set of attributes;

T: A set of data types (integer, real, string etc);

$\leq c$ : A partial order on $C$ called a hierarchy of concepts with a root named $c$;

$\leq R$ : a partial order on $R$, called the relationship hierarchy;

$\sigma R$ : A function called the relation signature;

$\sigma A$ : A function called the attribute signature;

We give a brief definition for each of these elements while adding instances and axioms.

1) Concept: The Iso standard [10] defines a concept as a "unit of knowledge created by a unique combination of characters". In general, a concept also called a class or entity represents in the ontological context a material object, a notion, etc [5].

2) Relation and signature of relationship " $R \& \sigma R$ ": Concepts can be interrelated by relationships within an ontology. The subsumption relation is-a defines a generalization link called hyperonymy and formally allows the inheritance of concepts. The is-a relation must be supplemented by other semantic relations to press out the semantics of the domain.

Consequently, a semantic relation $R$ is defined by the concepts $\left(c_{i}, c_{j}\right)$ that it connects with $c_{i}$ the domain of the relation and $c_{j}$ its co-domain or its range. These concepts constitute the semantic signature of the relation denoted by $\sigma R$. These relationships may have properties that may be algebraic properties (symmetry, reflexivity, transitivity) or cardinality properties [11].

3) Attribute and attribute signature " $A \& \sigma A^{\prime \prime}: \mathrm{A}$ concept can be characterized by valued properties, called attributes. For example, the person concept has the attributes name, first name and age.

The signature of the attribute $\sigma A$ is specified for a given attribute $A$ the concepts it describes and its data type. [5]

We also find:

4) Axioms (Differential Knowledge): Axioms are logical expressions used to merge concepts and relationships to define rules of inferences.

5) Instances (Individuals): They represent singular elements conveying the knowledge about the domain of the problem. 


\section{Editing ontology}

Many tools allow today to publish ontologies by guiding their user in the development of the ontology, while following a methodology of design, example the tool WebODE [10], Protege [12].

To build the ontology, the Protege environment is adopted. Protege [12] is a graphical ontology development environment developed by the University of Stanford based on the Methondology methodology [13] [14].

In Protege's knowledge model, ontologies consist of a hierarchy of classes that have attributes (slots), which can themselves have certain properties (facets). Editing these three types of objects is done through a graphical interface. The software architecture allows users to insert plugins that can bring new functionalities while benefiting from the latest advances in ontological research (knowledge base management, ontology visualization, alignment and merge).

\section{Exploitation of ontology}

An ontology allows the specification of knowledge thanks to an unambiguous formal language. Once created, the ontology cannot be used or exploited directly by the end-user, it is therefore necessary to represent it by means of a formalism which will acquire all its importance once integrated. The most prominent is the platform Jade and Jena Api.

1) Platform Jade: Jade [15] [16] (Java Agent Development Framework) is a multi-agent platform developed in Java by TILab S.p.A (Telecom Italia Lab). Its goal is to simplify the development of multi-agent systems by ensuring the conformity of the standards by a complete set of services and agents and by complying with the FIPA (Foundation for Intelligent Physical Agents) standards.

The exploitation of the ontology via Jade is done by a mapping process making it possible to obtain information in the format of object oriented language adapted for the agents Jade. But, this approach is rather tedious, and requires an expert in the field.

2) Jena Api: Jena [17] [18] is a Java Application Programming Interface (Api) for semantic web applications. It allows reading, manipulating ontologies described in RDFS or in OWL to apply certain mechanisms of inferences. Jena was developed by researchers in the UK (Hewlett-Packard) laboratories in the 2000s and has been widely used in semantic web applications.

The roles of Jena Api are varied, as:

- Manage ontologies (RDF-Schema, DAML + OIL, OWL) and make reasoning using knowledge of ontology.

- Facilitate the development of applications for the Semantic Web.

- $\quad$ Allow manipulation of RDF declarations.

- Allow RDF / XML reading and writing.
Knowing that this is a framework designed to operate ontologies, the Jena Api is adopted. This Api is an opensource project, including a set of programming tools, using the Java programming language.

\section{E. Degree of effectiveness of ontological relationships}

1) Notion degree of effectiveness: Once the relations extracted from the ontology, it is important to know their degree of effectiveness. We define this degree in production engineering enterprise level based on efficiency and effectiveness, in order to calculate the effectiveness of each relationship for performance improvement. Indeed, the two concepts of efficiency and effectiveness are among the classic evaluation criteria performances in the enterprise. According to [19], these two concepts are employed to resolve certain queries. Thus, a concept of efficiency the following issues : What material, human or financial resources have actually been mobilized? Are the effects accomplished with all the resources mobilized? Could we have attained the same results at a lower price?

The concept of effectiveness shows whether the intended outputs had they been implemented?

2) Concept of effectiveness degree: To assess the degree of effectiveness, we adopt the method of calculating the weighted average, well known and very popular in many areas- In multi-criteria decision analysis, the method is shown as a complete aggregation [20]. The principle of complete aggregation methods is to assess on a single measurement scale, the utility of each action in relation to each criterion. All of these utilities are then aggregated to obtain the full value of each share.

Knowing that we associate with each relationship extracted a type compared to the concepts of efficiency and effectiveness. Effectiveness for a type is measured as the ratio between the sum of the weighted values of this type and the total sum of the weighted values (formula (2)).

$$
\operatorname{Eff}_{\mathrm{typei}}=\frac{\sum_{1}^{2} w_{i}{ }^{*} \text { type }_{i}}{\sum_{1}^{2} w_{i}}
$$

The degree of effectiveness will be equal to half of the sum of effectiveness types (formula (3)).

$$
\text { DegreEff }=\frac{\sum_{1}^{2} E f f_{\text {type } i}}{2}
$$

\section{APPLICATION FOR A PRODUCTION ENTERPRISE}

We apply the proposed approach on a local manufacturing enterprise.

\section{A. Ontological modeling enterprise}

Following the proposed approach, the first step consists in the ontology building for the concerned enterprise. 
1) Enterprise specification: The enterprise chosen is a local production enterprise involved in the transformation wire drawing steel. The field of activity of the subsidiary is the production and sale of welded mesh panels, in rolls, concrete reinforcement and lattice girders.

The structural hierarchy of the enterprise consists of five departments (each of them contains sub-services) and two services:

- Commercial: This department defines trade policies to increase the company's sales and inventory management.

- Purchasing policies while managing the sales team to achieve the objectives.

- Supply: This department oversees procurement activities, inventory management and inventory management.

- Finance and accounting: This department takes part in the strategic decisions of the company while alerting the hierarchy on the possible risks, guarantees a financial balance, and optimizes the cash.

- Personnel and means: This department is responsible for the recruitment policy, skills management, human relations and social management of the subsidiary and the preservation of infrastructures and means.

- Technique: It defines the company's production and maintenance policy and ensures its implementation and follow-up.

The two services of the enterprise are:

- Industrial safety: This service advises and assists management in the development and organization of the safety policy (safety at work, working conditions) while ensuring the protection and preservation of the company's assets by reducing human and Materials.

- Management quality: This service sets up and organizes action plans to guarantee the quality of the company's products and ensures compliance with the normative requirements produced.

This hierarchy is governed by a deputy general director, finance and accounting manager, assistant for legal affairs, internal auditing of management, responsible for quality management, computer scientists and management control.

Based on our approach and after a site survey, we counted all interactions of the enterprise that we have split up into two parts: inter-enterprise interactions in number 9 and intra enterprise in number 24 .

The enterprise interacts with the outside world by nine entities: customer (client), supplier (fournisseur), bank (banque), port company (EPO), newspaper (journal), court (tribunal), prefecture (wilaya), contributions (cotisations) and reservations (reservations).

In this study, we focused on the interactions between the enterprise and the outside world.
2) Ontology adopted: Ontology has previously created and presents the interactions between the enterprise and the outside world. This ontology contains twenty-nine classes with nine relations. The nine relationships are:

- Relationship R_appro between Service of management stock and supplier.

- Relationship $R \_$achat between the Sales service and port company/ newspaper / bank / supplier.

- Relationship R_secu between Industrial safety service and prefecture.

- Relationship R_compta between Accounting service and customer / supplier/ bank.

- Relationship $R \_$manage between Chief of management quality and customer/ supplier.

- Relationship Appel_J between Legal businesses and customer / supplier / court.

- Relationship Ramene between Sales force and the customer.

- Relationship Reserve between the Service of general means and reservations.

- Relationship Cotise between the Chief of social relation and contributions.

Have worked with Protege [12] versions 3.4 and 4.1 Beta. After creation of ontology, owl file is generated and the plugin OntoViz [21] is used to show the hierarchy of classes. From the complete ontology (See Appendix A), part is selected to have good visibility. The Fig. 1. illustrates this hierarchy and interactions of supply department and management quality with the outside world.

With blue color represents all interactions of the two departments with outside world.

3) Reading ontology: After integration Api Jena in the Eclipse programming environment, we read the owl file generated from ontology in order to extract the relations.

We obtain a resulting set of Relations $R$ :

$\mathrm{R}=\left\{\mathrm{R}_{1}, \mathrm{R}_{2}, \ldots, \mathrm{R}_{\mathrm{n}}\right\}$, where each $\mathrm{R}_{\mathrm{i}}$ is related as a record with three arguments as the three criteria: $(\mathrm{S}, \mathrm{P}, \mathrm{O})$ with

$\mathrm{S}$ : Subject referring to a class or a relationship.

P: Property referring the type of subject.

This criterion can take three choices as $\mathrm{P}=\mathrm{P}[\mathrm{t}, \mathrm{d}, \mathrm{r}]$

With

t: type (relationship or class)

$\mathrm{d}$ : domain (input entity of the relationship)

r: range (output entity of the relationship).

$\mathrm{O}$ : An object that is a class.

We have the ability to generate all information of ontology namely classes and relationships or to filter based on the triple $\{\mathrm{S}, \mathrm{P}, \mathrm{O}\}$. Several combinations can be made, namely one, two or three filters.

Our goal consists in extracting all relationships, so we take as a filter criterion $\mathrm{P}$ (property) with the choice of (domain). We will have as output correspondence, relationships and classes of the kind:

Relationship / domain / Class name.

For example, Fig. 2 lists for any relationship the incoming classes (domains). 
According to the selected departments (Fig. 1), we have as a result for supply department two domains:

\section{R_appro | domain | S_gestion_stock (Service of} management stock)
R_achat | domain | S_gestion_stock (Service of management stock)

For management quality, we have one domain:

$R \_$manage $\mid$domain $\mid$responsible_management_ qualite (Chief of management quality)

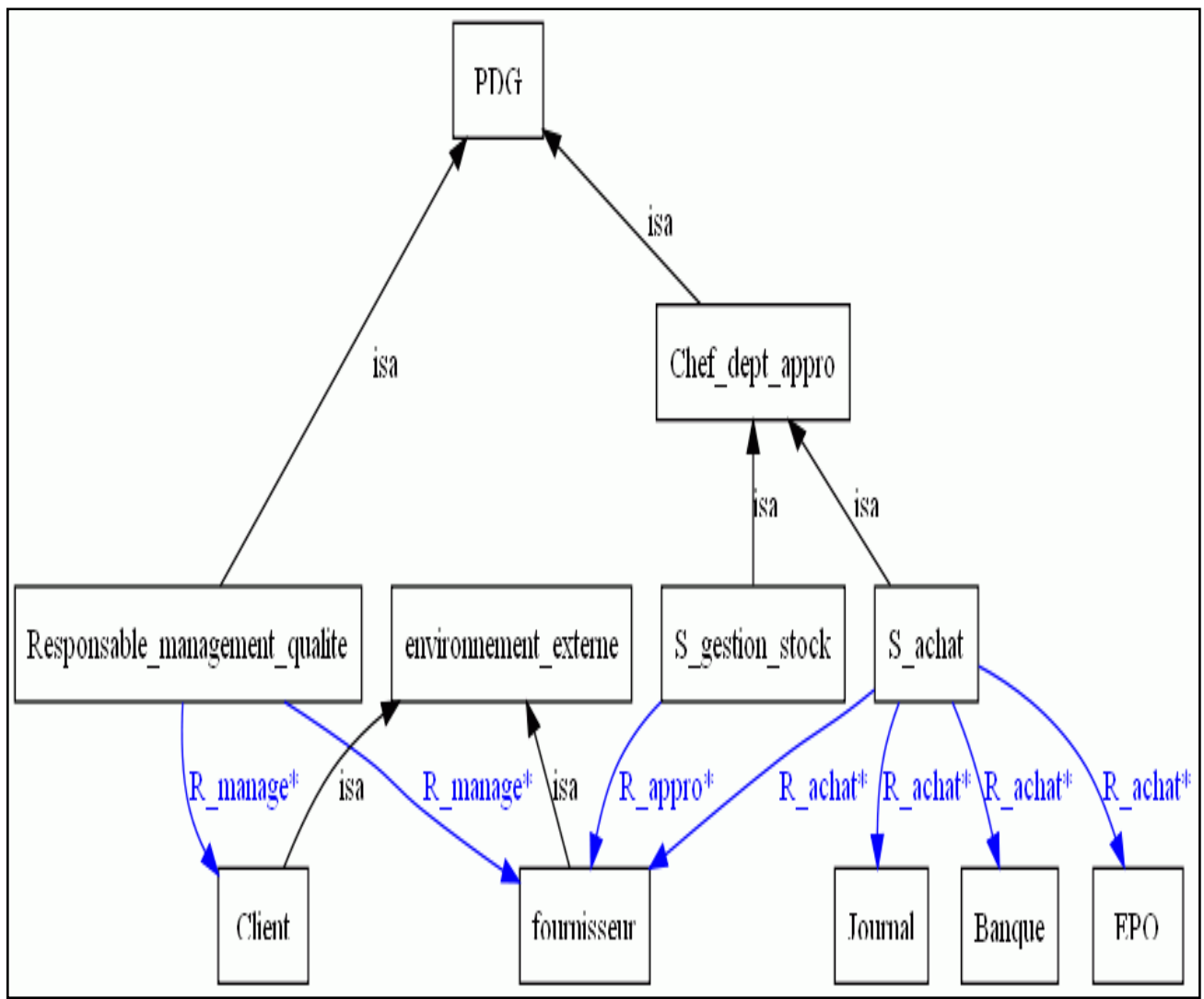

Fig.1. Interactions of supply department and management quality with outside world

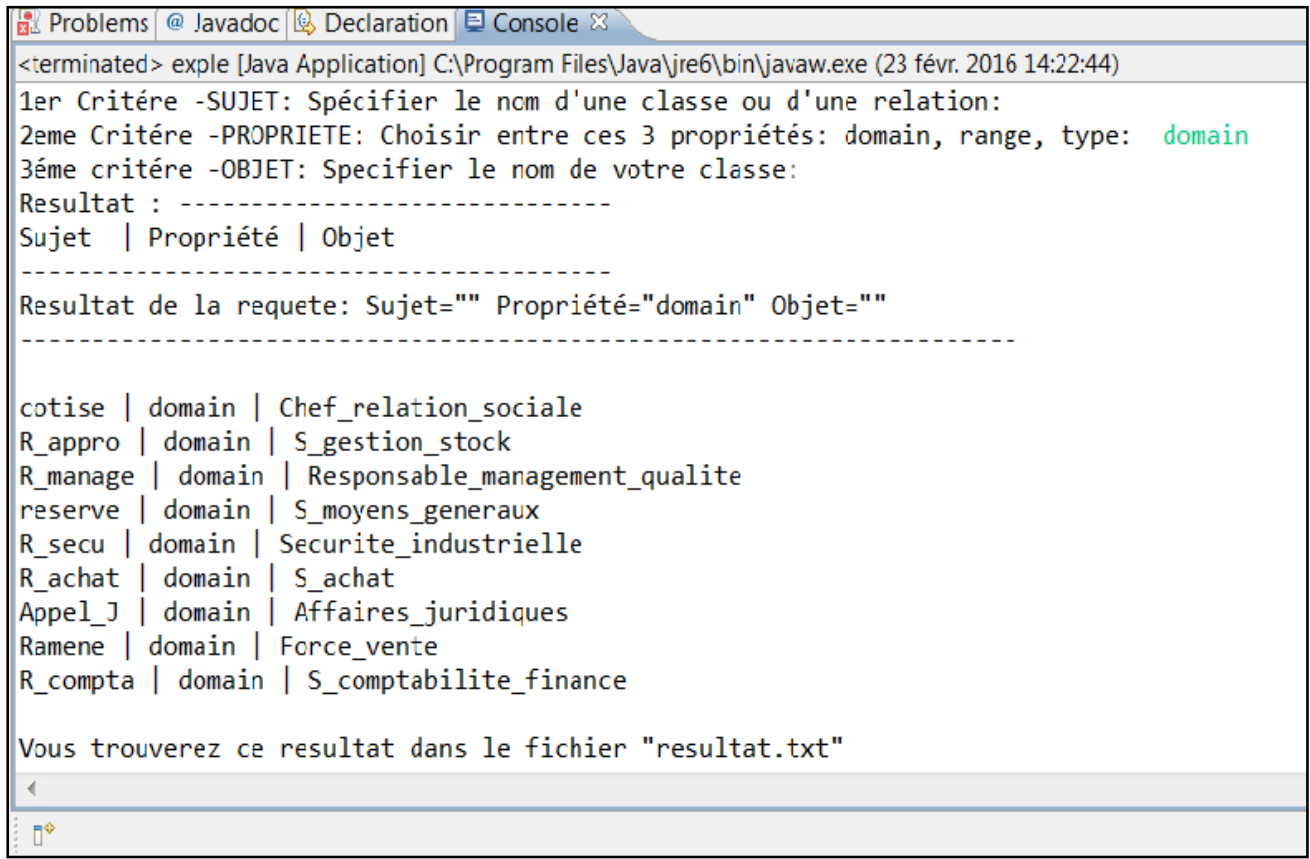

Fig.2. Domains of enterprise relationships 


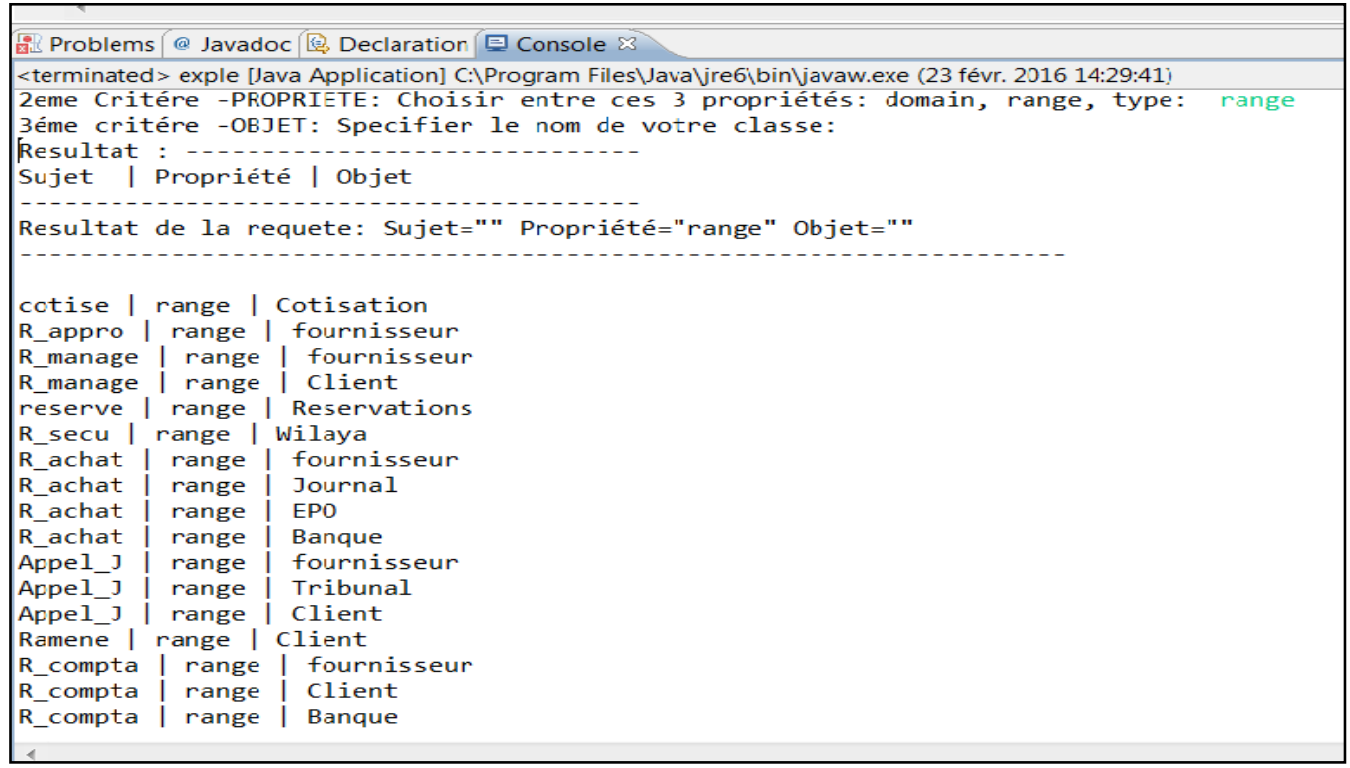

Fig.3. Ranges of the enterprise's relationships

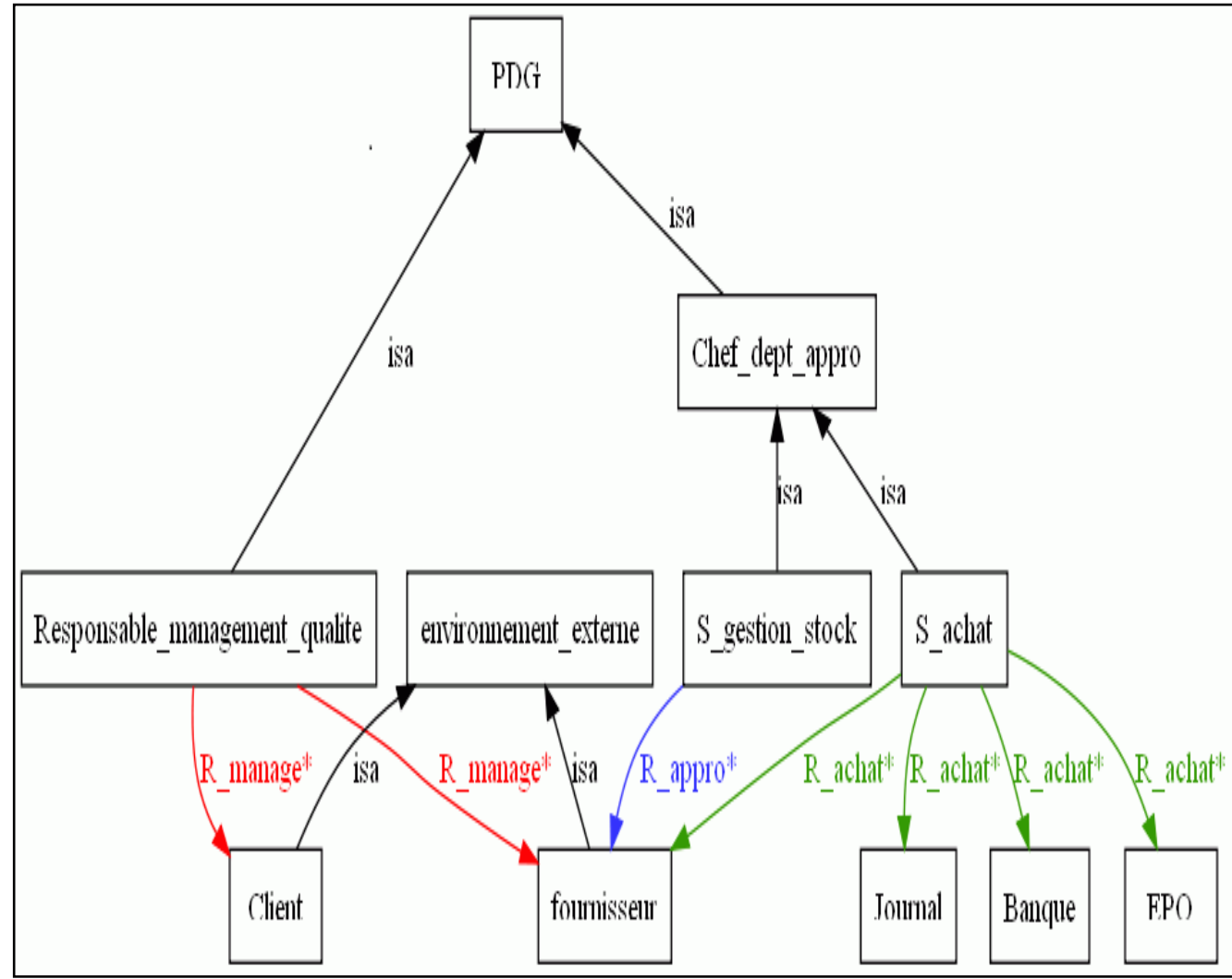

Fig.4. Weighted interactions of the supply department and management quality with outside world

This result illustrates the generation of input entities of each relationship. Following the same process, we generate the output entities by replacing domain range (Fig.3)

- Relationship $R \_a c h a t$ between the Sales service and port company/ newspaper / bank / supplier satisfying both of efficiency and effectiveness.
- Relationship R_compta between Accounting service and customer / supplier/ bank satisfying both of efficiency and effectiveness.

- Relationship R_manage between Chief of management quality and customer/ supplier satisfying effectiveness.

- Relationship Appel_J between Legal businesses and customer / supplier / court satisfying effectiveness. 
- Relationship Ramene between Sales force and the customer satisfying effectiveness.

- Relationship Reserve between the Service of general means and reservations satisfying efficiency.

- Relationship Cotise between the Chief of social relation and contributions satisfying efficiency.

Once weighted relations, we take the ontology creates and generate via the plugin OntoViz [22] all weighted extracted interactions (see Appendix B).

The Fig. 4 illustrates the weighted relations of supply department and management quality with outside world.

With

Blue color Relationship satisfying efficiency.

Red color: Satisfactory relationship effectiveness.

Green color: Relationship satisfying both of efficiency and of effectiveness.

\section{B. Measuring the effectiveness of weighted Relations}

1) Weighting relations: In order to improve enterprise performance and following the approach, we classify the relationships extracted from the ontology following the two types that is efficiency and effectiveness. Since the chosen enterprise is a production enterprise, we put a weight to

2) effectiveness type that is a doubled measure in comparison with efficiency type.

So we get for the type efficiency weighing 0.33 and effectiveness weighing 0.67 (the sum of the weight is 1). Table 1. summarizes the weights of the identified relationships.

Table 1. Weighting of semantic relations

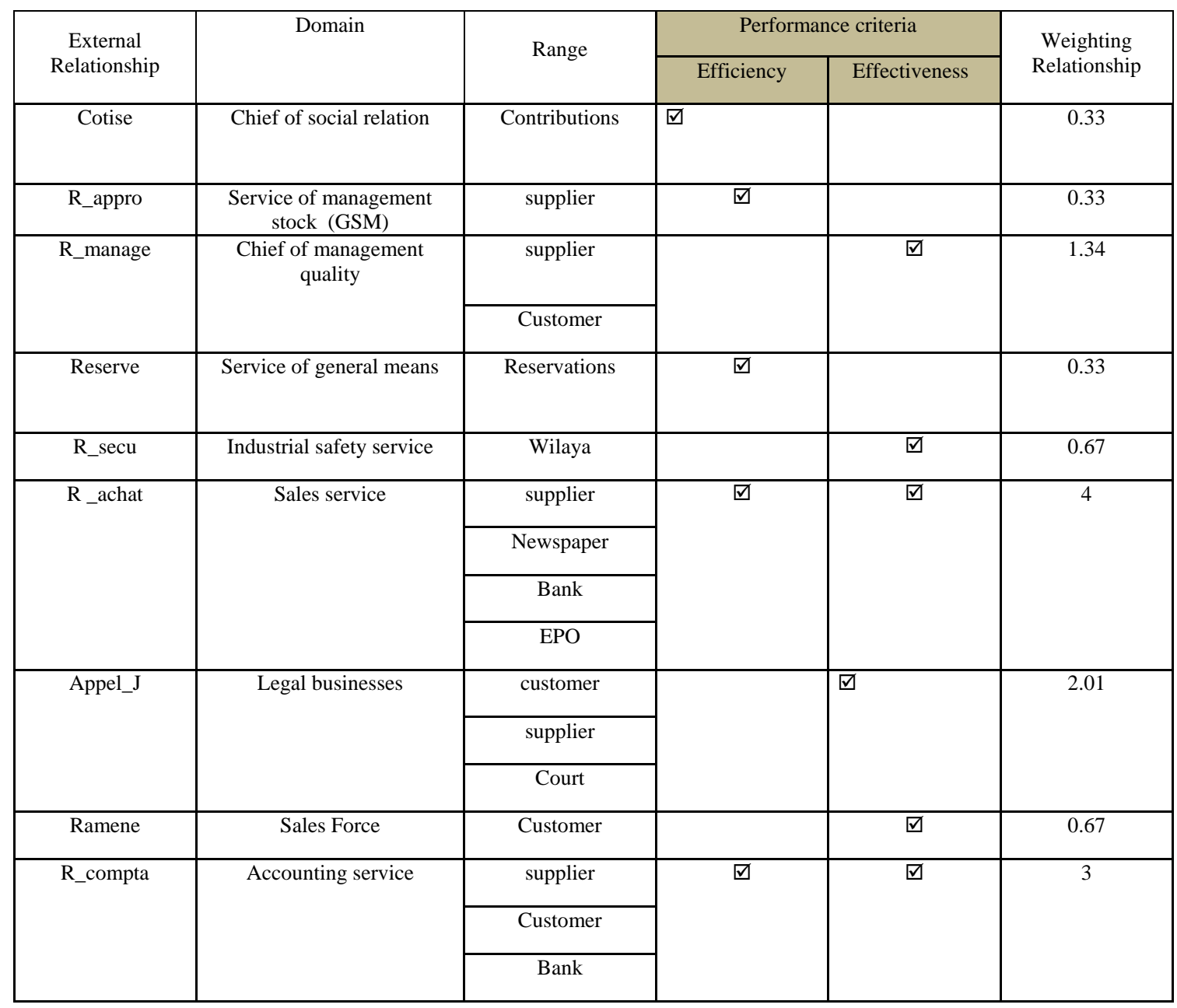

From the table, an histogram (Fig. 5) is presented for the weighting of the company relationship.

The Purchasing relationship is the biggest weighting and it's justified because our work focused on the company's interactions with the outside world and the purchasing department of the Procurement department interacts with the majority of entities in the external world. 


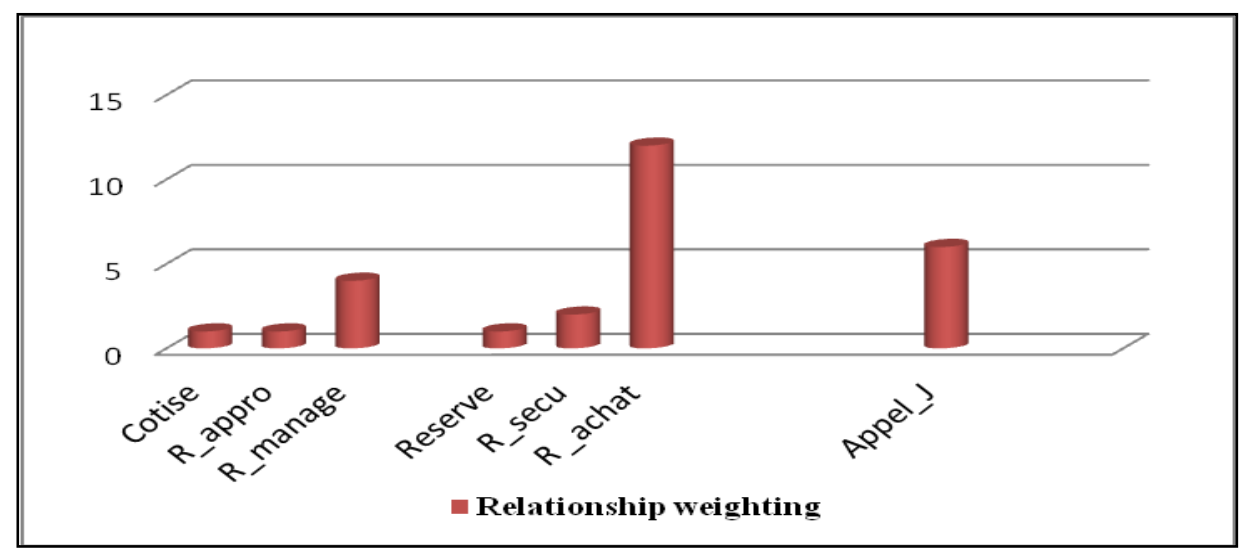

Fig.5. Relationship weighting graph

3) Calculation of the degree of effectiveness: We calculate the degree of effectiveness of the first type (Efficiency type) and the second type Effectiveness type) according to the formula (2). Recall that formula (2) calculate the effectiveness relative to a type and is measured as the quotient between the sum of the weighted values of this type and the total sum of the weighted values. We get:

- $\quad$ Eff $_{\text {Typel }}=0.630$

- $\quad$ Eff $_{\text {type } 2}=0922$

We calculate the average of the degrees of effectiveness following formula (3) we get: DegreEff = $78 \%$

\section{Sensitivity analysis}

A sensitivity analysis is proposed with two experiments, established on the variation of weight, to see if the result is robust to slightly different preferences, or even very different.

\section{1) The two experiments}

- First experiment: Knowing that the weighting chosen respects the fact that the sum is equal to 1 , we slightly vary these weights on the interval $[0.29,0.34]$ and $[0.58,0.68]$ and recalculate for a sensitivity study.

By assigning a weight of 0.34 to the type of efficiency (type 1) and 0.68 to the type of effectiveness (Type 2), we get, following formula (2) : Eff $f_{\text {Type } 1}=0.632$ and Eff ${ }_{\text {Type } 2}=$ 0.921

We calculate the average of the degrees of effectiveness following formula (3) we will always have DegreEff $=78 \%$

- Second experiment: We assign a weight to the type effectiveness (type 2) by contribution equal to three times the type efficiency (type 1). The weight of the type effectiveness will be 0,75 and for the type efficiency will be 0.25 . By following formula (2) we get : Eff $_{\text {Type } 1}=0.596$ and Eff $_{\text {Type2 }}=$ 0.942

We calculate the average of the degrees of effectiveness following formula (3), we will have a DegreEff $=77 \%$

- Interpretation of results: From the previous sensitivity analysis, we conclude that the change in weight has little impact on the weighted average since the difference is negligible. To validate this result on the degree of effectiveness (DegreEff = $77 \%$ or $78 \%$ ), we calculate the effectiveness of semantic relationships extracted following formula (1) and we get: Eff $_{\text {RelSem }}=9 / 17=53 \%$.

This rate is significantly lower than the rate of effectiveness weighted relationships. This result is justified by the fact that we have carried out our calculations taking into account in a weighted relationship two important criteria in a real production enterprise.

\section{CONCLUSION}

We have presented in this article measures for ontological relations in enterprise. We discussed the notion of effectiveness of semantic relationships while detailing the proposed approach. Our goal was to be able to calculate the actual degree of interaction in the enterprise. The case study was made on a corporate real example of transformation and drawing of steel. Following this study, we classified the relationships extracted from an ontology according to their relative weight to efficiency and effectiveness in a context of production. Following this weight, we calculated the degree of effectiveness of the relationship. On the other hand, we followed a calculation of effectiveness of semantic relations and by adding the type efficiency that indicates whether the results obtained are consistent with the targets set up. 


\section{APPENDIX}

Appendix A: All of the enterprise's interactions with the outside world

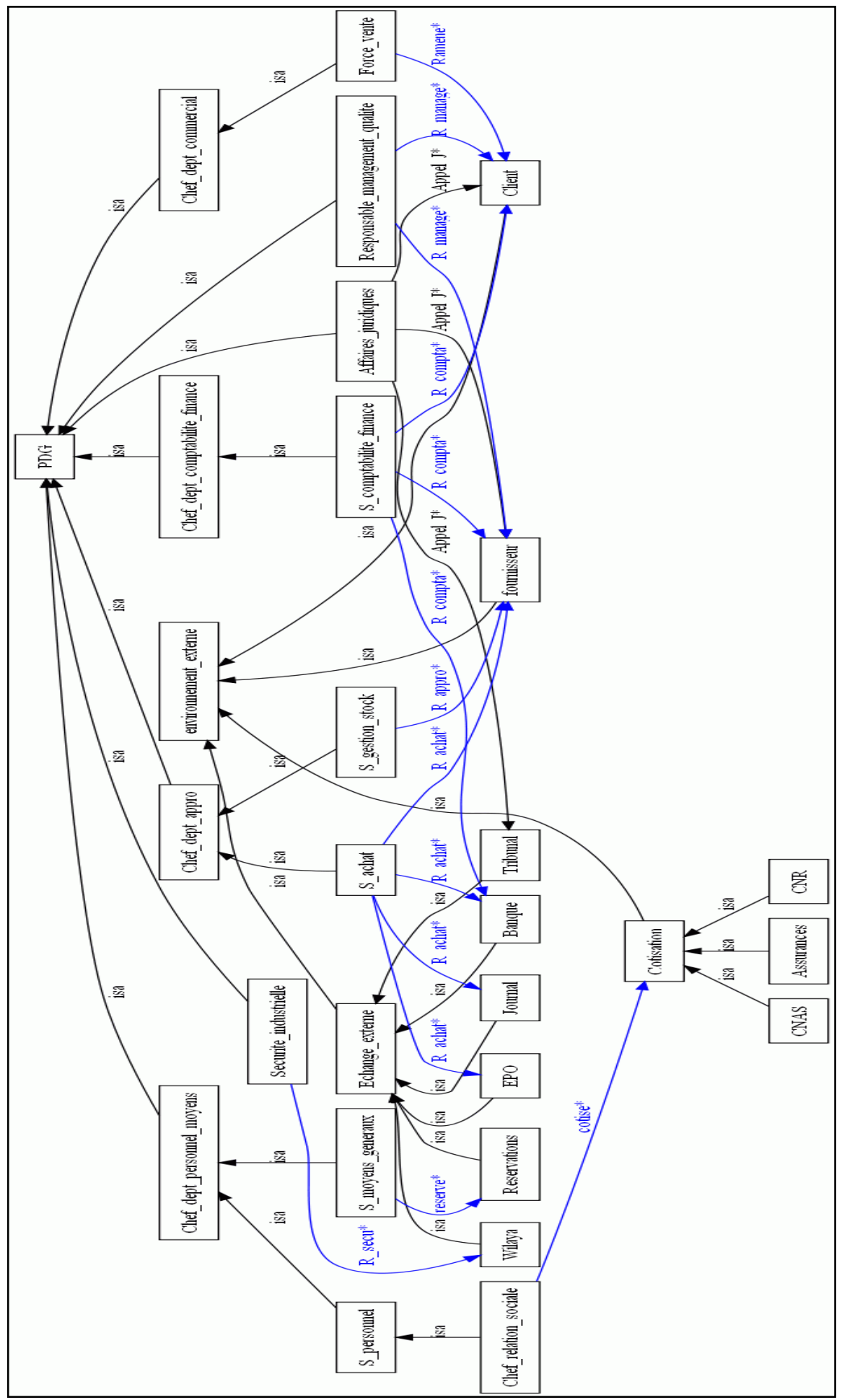


Appendix B: Weighted ontology

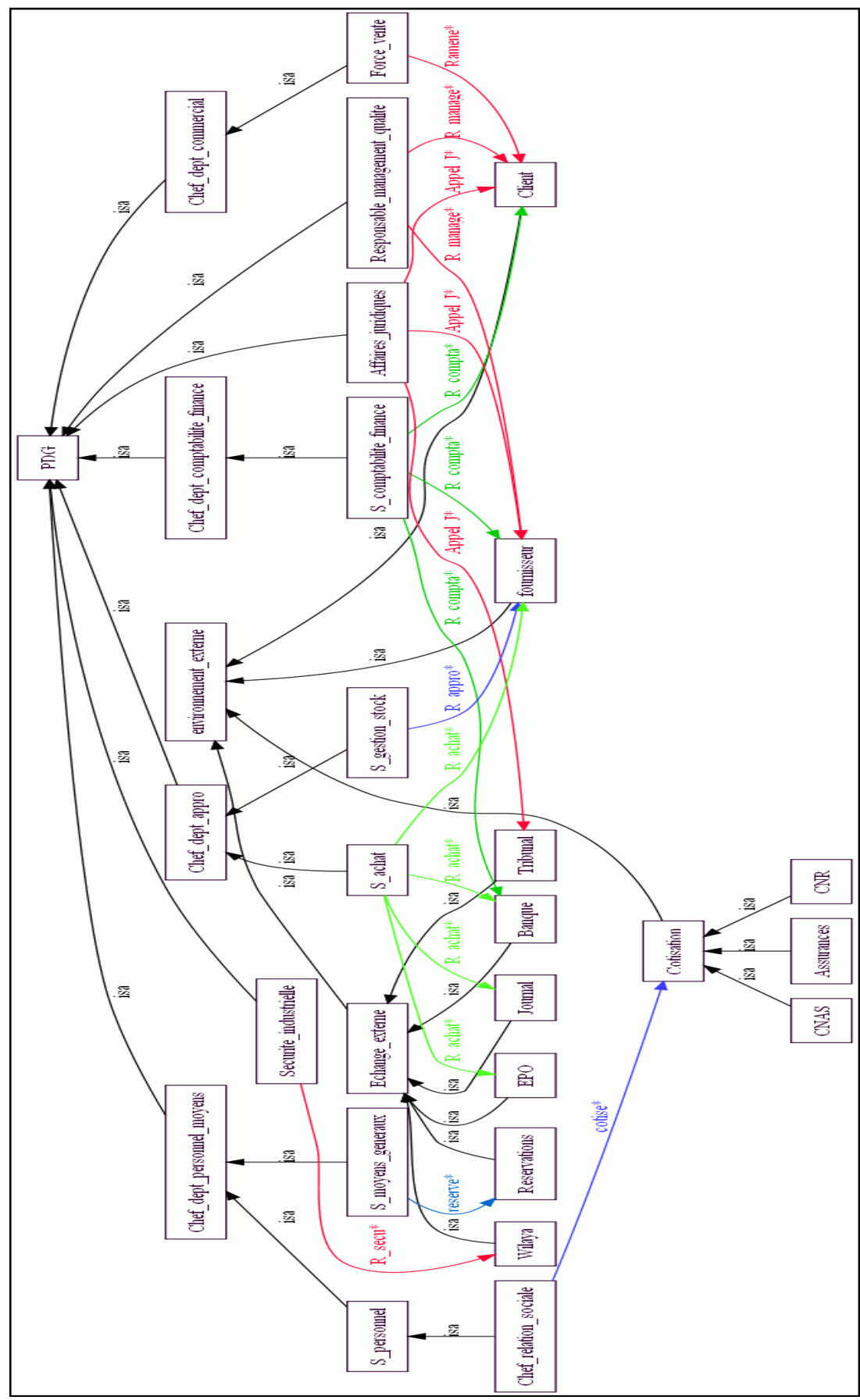

With blue color represents all enterprise's interactions with outside world.

With:

Blue color: Relationship satisfying efficiency.

Red color: Satisfactory relationship effectiveness.

Green color: Relationship satisfying the efficiency and effectiveness 


\section{REFERENCES}

[1] D. Chen, G. Doumeingts, F Vernadat "Architectures for enterprise integration and interoperability: Past, present and future" . Journal of Computers in industry, Elsevier, pp.647-659, 2008.

[2] N. Mellal. "Réalisation de l'interopérabilité sémantique des systèmes, basée sur les ontologies et les flux d'information ". Doctoral thesis, University of Savoie, 2007.

[3] JL. Moigne. "Modeling complex systems". Dunod, 1990.

[4] E. Yahia, M. Lezoche, A. Aubry, H. Panetto. "Semantics enactment for interoperability assessment in enterprise information systems". Annual Reviews in Control 36Elsevier, pp. 101-117,2012.

[5] T. Gruber. "A translation approach to portable ontology specifications". Knowledge Acquisition, pp. 199-220, 1993.

[6] F. Fürst. "Contribution à l'ingénierie des ontologies : une méthode et un outil d'opérationnalisation". Doctoral thesis, EPU-Nancy (France), 2004.

[7] U.Yadav, GS Narula, N.Duhan, V.Jain. "Ontology Engineering and development aspects: A survey". International Journal of Education and Management Engineering JEME, 2016.

[8] RJ. Rovetto. " An ontological architecture for orbital debris data", Earth science informatics, Springer, USA, 2016.

[9] P. Cimiano. "Ontology learning and population from text algorithms, evaluation and applications".Springer Science \& Business Media, 2006.

[10] Iso 1087. "Terminology work-vocabulary , theory and application first edition". Licensed to Business semantics LTD/Donald chapin, 2000.

[11] J. Arpirez , O. Corcho, M. Fernández-López, A. GómezPérez A. "WebODE : a workbench for ontological engineering". First international Conference on Knowledge Capture, pp.6-13, 2001.

[12] Tutorial tool Protege http://protege.stanford.edu/

[13] M. Fernandez-Lopez, A. Gomez-Perez, N. Juristo. "Methontology: from ontological art towards ontological engineering". Proceedings of the AAAI97 Springer Symposium, pp. 33-40, Stanford, USA, 1997.
[14] O. Corcho, A. Fernandez-Lopez, A. Gomez-Perez A. Lopez-Cima. "Building legal ontologies with methontology and WebODE". Law and the Semantic Web, vol. 3369, Springer, pp.142-157, 2005.

[15] B. Mustafa, N. Aldabagh. "Ojadeac, an ontology based access control model for jade platform". International Journal of Advanced Computer Science and Applications, Vol. 5, No. 5. 2014.

[16] Official site of JADE http://jade.tilab.com

[17] M. Horridge, S. Bechhofer. "The owl api: a java api for owl ontologies". Journal Semantic Web. Volume 2 Issue 1, pp.11-21. 2011.

[18] Official site of JENA http://jena.sourceforge.net/

[19] V. Galdemar, L. Gilles, MO. Simon. " Performance, efficacité, efficience : les critéres d'evaluation des politiques sociales sont-ils pertinents ?", CREDOC, research paper number 209, 2012.

[20] S.Greco, B.Matarazzo, R.Slowinski. "Rough sets theory multicriteria decision analysis". European Journal of Operational Research, elsevier, 2001.

[21] Tutorial plugin OntoViz. http://protegewiki.stanford.edu/wiki/OntoViz

\section{Author's profiles}

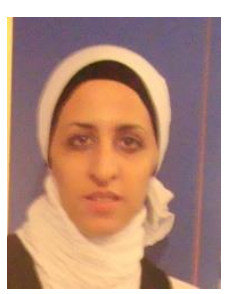

Hadj Tayeb Sabria: $\mathrm{PhD}$ student in Computer Science. Currently is Assistant Professor at the Height school in Electrical and Energy Engineering in Oran (ESGEE). Her research interests are in the field of ontology and interoperability in enterprises.

Myriam Noureddine is currently Assistant Professor in the department of computer science in the University of Sciences and Technology of Oran (USTO). Her research interests focus on the modeling methods, dependability domain and the performance improvement of industrial enterprises.

How to cite this paper: Sabria Hadj Tayeb, Myriam Noureddine," Measures for the Ontological Relations In Enterprise", International Journal of Modern Education and Computer Science(IJMECS), Vol.9, No.9, pp. 13-23, 2017.DOI: $10.5815 /$ ijmecs.2017.09.02 